\section{S98 ANTACID THERAPY AND DISEASE PROGRESSION IN PATIENTS WITH IDIOPATHIC PULMONARY FIBROSIS (IPF) UNDER PIRFENIDONE TREATMENT}

\begin{abstract}
${ }^{1} \mathrm{M}$ Kreuter, ${ }^{2} \mathrm{P}$ Spagnolo, ${ }^{3} \mathrm{~W}$ Wuyts, ${ }^{4} \mathrm{E}$ Renzoni, ${ }^{5} \mathrm{D}$ Koschel, ${ }^{6} \mathrm{~F}$ Bonella, ${ }^{4} \mathrm{TM}$ Maher, ${ }^{7} \mathrm{M}$ Kolb, ${ }^{8} \mathrm{D}$ Weycker, ${ }^{9} \mathrm{~K}$ Kirchgässler, ${ }^{6} \mathrm{U}$ Costabel. ${ }^{1}$ Pneumology and Respiratory Critical Care Medicine, Centre for Interstitial and Rare Lung Diseases, Thoraxklinik, University of Heidelberg, Heidelberg, Germany and Translational Lung Research Centre Heidelberg (TLRCH), German Centre for Lung Research (DZL), Heidelberg, Germany; ${ }^{2}$ Section of Respiratory Diseases; Department of Cardiac, Thoracic and Vascular Sciences; University of Padua, Padua, Italy; ${ }^{3}$ Unit for Interstitial Lung Diseases, Department of Respiratory Medicine, University Hospitals, Leuven, Belgium; ${ }^{4}$ National Institute for Health Biomedical Research Unit, Royal Brompton Hospital and National Heart and Lung Institute Imperial College, London, UK; ${ }^{5}$ Department of Pulmonary Diseases, Fachkrankenhaus Coswig, Centre for Pulmonary Diseases and Thoracic Surgery, Coswig, Germany; ${ }^{6}$ Interstitial and Rare Lung Disease Unit, Ruhrlandklinik, University Hospital, University of Duisburg-Essen, Essen, Germany; ${ }^{7}$ Firestone Institute for Respiratory Health, Department of Medicine, Pathology and Molecular Medicine, McMaster University, Hamilton, Ontario, Canada; ${ }^{8}$ Policy Analysis Inc. (PAI), MINERVA Health Economics Network, Ltd., Brookline, MA, USA; ${ }^{9} \mathrm{~F}$. Hoffmann-La Roche Ltd, Basel, Switzerland
\end{abstract}

\subsection{6/thoraxjnl-2016-209333.104}

Introduction On the basis of retrospective and post-hoc analyses the current IPF guideline suggests the use of anti-acid therapy (AAT, i.e., proton pump inhibitors and H2-blockers) as a treatment option in patients with IPF. While recent post-hoc analyses do not support a protective effect of AAT on IPF progression in patients receiving placebo, the impact of AAT on disease progression in patients treated with pirfenidone is unknown.

Methods Patients with IPF randomised to pirfenidone in 3 trials (CAPACITY studies 004 and 006, and ASCEND) were included. Changes in pulmonary function, exercise tolerance, survival, hospitalizations, and adverse events (AEs) over 52 weeks were analysed for all subjects, based on AAT status at baseline, by bivariate and multivariate analyses. Disease progression was defined as an absolute decrease of forced vital capacity (FVC) $\geq 10 \%$ predicted, a decrease of $\geq 50 \mathrm{~m}$ in the 6 -minute walk distance (6MWD) or death.

Results Of 623 patients, 44\% received AAT. Patient characteristics were comparable between groups with the exception of gastrointestinal (GI) comorbidities. In bivariate analyses, there were no significant differences at 52 weeks in disease progression (AAT vs non-AAT: $24.9 \%$ vs $30.6 \% ; P=0.12$ ), all-cause or IPFrelated mortality $(2.9 \%$ vs $4.0 \% ; P=0.47$ and $1.1 \%$ vs $2.0 \%$; $P=0.37$, respectively), all-cause hospitalisation $(16.1 \%$ vs. $18.3 \%, P=0.48)$ or observed mean FVC decline $(-2.7 \%$ vs $-3.1 \%, P=0.44)$. Relative but not absolute FVC decline $\geq 10 \%$ was slightly in favour of AAT $(15 \%$ vs $22 \% ; P=0.03)$. In multivariate analyses, hazard ratios across study outcomes ranged from 0.3-0.9 for AAT (vs. non-AAT), although differences were not statistically significant (including relative FVC decline $\geq 10 \%$ ). AEs were generally similar between groups; however, severe GI AEs $(3.7 \%$ vs. $0.9 \%, P=0.015)$ and severe pulmonary infections (3.7\% vs. $1.1 \%, P=0.035)$ were more frequent in AAT users. Conclusion In this post-hoc analysis of three randomized-controlled trials, there was no clear evidence of benefit of the combination of AAT and pirfenidone compared to pirfenidone alone. However, AAT use appeared to increase the risk of severe GI and infectious AEs. AAT should be prospectively assessed in a randomised controlled trial before being considered as a specific treatment for IPF. REPORTED AS SERIOUS ADVERSE EVENTS IN THE INPULSIS ${ }^{\circledR}$ TRIALS IN IDIOPATHIC PULMONARY FIBROSIS (IPF)

${ }^{1} \mathrm{~L}$ Richeldi, ${ }^{2} \mathrm{H}$ Koegler, ${ }^{2} \mathrm{M}$ Trampisch, ${ }^{2} \mathrm{~S}$ Geier, ${ }^{3} \mathrm{M}$ Kreuter. ${ }^{1}$ National Institute for Health Research Southampton Respiratory Biomedical Research Unit and Clinical and Experimental Sciences, University of Southampton, Southampton, UK; ${ }^{2}$ Boehringer Ingelheim Pharma $\mathrm{GmbH}$ and Co. KG, Ingelheim am Rhein, Germany; ${ }^{3}$ Centre for Interstitial and Rare Lung Diseases, Department of Pneumology, Thoraxklinik, University of Heidelberg, and Translational Lung Research Centre Heidelberg, German Centre for Lung Research Germany, Heidelberg, Germany

\subsection{6/thoraxjnl-2016-209333.105}

Introduction The INPULSIS ${ }^{\circledR}$ trials assessed the effects of nintedanib versus placebo in patients with IPF. Time to first investigator-reported acute exacerbation over 52 weeks was a key secondary endpoint. Adverse events that were considered by an investigator to fulfil pre-defined criteria for an acute exacerbation were categorised by an adjudication committee as a confirmed acute exacerbation, suspected acute exacerbation, or not an acute exacerbation. We assessed the effect of nintedanib on acute exacerbations reported as serious adverse events and non-serious adverse events and the impact of these events on survival.

Methods A post-hoc analysis of patients with acute exacerbations reported as serious adverse events or non-serious adverse events over 52 weeks was undertaken using pooled data from the INPULSIS ${ }^{\circledR}$ trials.

Results Of the 63 patients who had $\geq 1$ investigator-reported acute exacerbation, $49(77.8 \%)$ had an acute exacerbation reported as a serious adverse event. Of these 49 patients, 31 (63.3\%) had an adjudicated confirmed or suspected acute exacerbation reported as a serious adverse event. A higher proportion of patients with investigator-reported acute exacerbations reported as serious adverse events died than patients with acute exacerbations reported as non-serious adverse events (30 of 49 patients [61.2\%] versus 1 of 15 patients [6.7\%]) (Figure). Nintedanib significantly reduced the risk of a first investigator-reported acute exacerbation reported as a serious adverse event versus placebo (HR 0.57 [95\% CI: 0.32, 0.99]; p = 0.0476). Investigatorreported acute exacerbations reported as serious adverse events occurred in $3.6 \%$ of patients in the nintedanib group and $6.1 \%$ in the placebo group. Nintedanib significantly reduced the risk of having a first adjudicated confirmed or suspected acute exacerbation reported as a serious adverse event versus placebo (HR 0.30 [95\% CI: 0.14, 0.64]; $\mathrm{p}=0.0019)$. Adjudicated confirmed or suspected acute exacerbations reported as serious adverse events occurred in $1.6 \%$ in the nintedanib group and $5.0 \%$ in the placebo group.

Conclusion In pooled data from the INPULSIS ${ }^{\circledR}$ trials, nintedanib significantly reduced the risk of acute exacerbations reported as serious adverse events. Acute exacerbations reported as serious adverse events were associated with a much higher risk of death than acute exacerbations reported as non-serious adverse events. 